\title{
Bilateral Ageusia and Tongue Anesthesia Following Unilateral Brainstem Infarct: A Case Report with a Brief Review of the Literature
}

\author{
Christian Saleh $^{a}$ Simona Negoias ${ }^{b}$ Franca Wagner $^{c}$ Marie- \\ Luise Mono ${ }^{a}$ \\ ${ }^{a}$ Department of Neurology, Inselspital, Bern University Hospital and University of Bern, \\ Bern, Switzerland; 'bepartment of Otorhinolaryngology-Head and Neck Surgery, \\ Inselspital, Bern University Hospital, University of Bern, Bern, Switzerland; ' Institute of \\ Diagnostic and Interventional Neuroradiology, Inselspital, University Hospital Bern and \\ University of Bern, Bern, Switzerland
}

\section{Keywords}

Stroke $\cdot$ Ageusia $\cdot$ Weight loss

\section{Abstract}

We present the case of a 63-year-old male patient who suffered bilateral ageusia following a unilateral left-sided mesencephalon infarct. To the best of our knowledge, this is the first description of a mesencephalon lesion leading to ageusia. We discuss the literature on this rare but important symptom following a stroke.

\section{Introduction}

Loss of taste (ageusia) is a rare consequence of stroke and can present as its sole clinical finding. Ageusia may be considered at first a clinical deficit of lesser severity compared to classic stroke symptoms such as hemiparesis, vision deficit, vertigo or language disorders (aphasia, dysarthria). However, ageusia can have serious clinical consequences considering 
its close relationship to the subject's quality of life. Depression, malnutrition, and weight loss can be short- or long-term complications [1]. Brainstem infarcts (pons) and supratentorial infarcts (thalamus and insular cortex) may be associated with ageusia. We report the case of a patient with a unilateral mesencephalic infarct who suffered bilateral ageusia with tongue anesthesia. To the best of our knowledge, this is the first description of a unilateral mesencephalon lesion causing a bilateral ageusia and tongue anesthesia. Our case report is supplemented by a brief review of the literature discussing the relationship between stroke and ageusia.

\section{Case Report}

A 63-year-old male patient suffered a few-second-lasting frontal headache with instant loss of any taste perception and entire tongue numbness while having lunch. He denied any weakness, paresthesia, vision or speech disturbances. On admission, his National Institutes of Health Stroke Scale (NIHSS) score was 1 (for tongue anesthesia). Vital signs were within normal range, except for an elevated blood pressure. He had a previous history of recurrent vertebro-basilar cerebrovascular accidents and stent implantation in the left vertebral artery (at the level of the ostium). Vascular risk factors were hypertension, hypertensive cardiomyopathy, coronary artery disease with percutaneous transluminal coronary angioplasty, diabetes mellitus, nicotine abuse, dyslipidemia, and a light sleep apnea syndrome. He had a history of left ventricular thrombus and atrial fibrillation successfully treated with cardioversion in the past. A cardioverter defibrillator had been implanted in 2012 (Model Medtronic Protecta XT), which was not MRI compatible. Medication on admission was aspirin $100 \mathrm{mg}$ once daily, metoprolol $25 \mathrm{mg}$ once daily, amiodarone $200 \mathrm{mg}$ once daily, apixaban $2.5 \mathrm{mg}$ twice daily, empagliflozin $10 \mathrm{mg}$ once daily, metformin $500 \mathrm{mg}$ twice daily, atorvastatin $40 \mathrm{mg}$ once daily, and perindopril $10 \mathrm{mg}$ once daily.

Blood examination was normal for electrolyte, kidney, liver function, cholesterol, and cardiac enzymes; leukocytes were mildly raised $(12.6 \mathrm{~g} / \mathrm{L})$ as well as neutrophil count (8.7 $\mathrm{g} / \mathrm{L}$ ) with a normal C-reactive protein level. The patient did not show any clinical signs of infection. $\mathrm{HbA}_{1 \mathrm{c}}$ was elevated at $9.3 \%$.

CT imaging on admission day (externally acquired noncontrast CT with CT angiography) revealed a known severe leukoencephalopathy and chronic lacunar defect at the level of the right thalamus and the left precentral frontal region. There were no signs of intracranial hemorrhage or acute/subacute infarction. The brain-supplying arteries were unremarkable.

The follow-up CT 1 day after the patient's transfer to our university hospital revealed a subtle oval hypodensity located on the left side in the mesencephalon (Fig. 1) with associated perfusion deficit (Fig. 2), reduced cerebral blood flow (Fig. 2a) and cerebral blood volume (Fig. 2b) with prolonged mean transit time (Fig. 3a), time to drain (Fig. 3b) and time to peak (Fig. 3c) indicative of a subacute focal brainstem ischemia.

Ultrasound of the brain-supplying vessels showed a mild flow acceleration at the level of the left vertebral artery (at stent level) but no significant stenosis. Cardiac workup (electrocardiogram and transesophageal echocardiography) was normal.

To optimize secondary prophylaxis, we increased the dose of apixaban from $2.5 \mathrm{mg}$ twice daily to $5 \mathrm{mg}$ twice daily. Aspirin $100 \mathrm{mg}$ once daily was changed to clopidogrel $75 \mathrm{mg}$ once daily to increase the platelet anti-aggregation effect in light of atherosclerotic progression in the left vertebral artery. 
Atorvastatin was increased from $40 \mathrm{mg}$ once daily to $80 \mathrm{mg}$ once daily to enhance plaque stability. We hypothesized as cause of the stroke an embolic event originating from the left stenotic vertebral artery ipsilateral to the subacute mesencephalic infarction area.

During his 2-day hospitalization, the patient did not develop any further neurological deficit. Screening for taste and smell on admission did not show any loss of smell function but a complete and bilateral loss of taste perception with tongue anesthesia.

Taste function was evaluated on follow-up examination at 2 months with the standardized "Taste Strips" battery (Taste Strips, Burghart, Wedel, Germany) [2]. Results showed a bilateral ageusia with an overall score of $8 / 32$ identified taste strips $(3 / 16$ for the left side and $5 / 16$ for the right side). Smell testing was performed with the screening subtest of the standardized Sniffin' Sticks test battery [3, 4], consisting of a 12-item identification test. Results showed a normosmia with 10 out of 12 correctly identified items. Self-evaluation of taste and smell function as well as level of life quality impairment through the dysfunction were recorded on visual analogue scales ranging from 0 (very low level of smell, taste function or impairment of life quality) to 100 (very good smell and taste function, very high level of life quality impairment). The patient reported his taste function with $2 / 100$, his smell function with 73/100, and his life quality impairment with $97 / 100$. He complained of persistent tongue anesthesia with loss of appetite and enjoyment of eating and cooking. No ear symptoms or history of ear surgery were reported and the ear examination showed no pathological findings.

\section{Discussion}

The anatomic gustatory pathway starts with afferents from the facial, glossopharyngeal, and vagus nerves, which project on the solitary tract nucleus located at the medulla. The gustatory lemniscus ascends ipsilaterally to the pons. Current data suggest that gustatory fibers may decussate at the mesencephalon and project on the ventroposteromedial nucleus and dorsomedial thalamic nuclei terminating at the primary taste cortex located in the frontal operculum and insular cortex with the secondary cortical taste area located at the orbitofrontal cortex [5]. However, the gustatory pathway is not yet fully elucidated. Multiple lesion locations were related to ageusia, foremost the insular cortex [6-10], the pons $[5,11$, $12]$, and the thalamus [13].

\section{Insular Cortex}

Several diverse taste alterations were reported with left insular infarct [6-8] and rightsided insular lesions [8-10]. A patient suffered changes for salty and sour taste recognition with normal recognition for bitter and sweet following stroke of the left insular cortex [6], while in another patient the same lesion location resulted in a diminished pleasure in taste and persistent taste of rotten melon [7]. An ipsilateral deficit in taste intensity, but a bilateral deficit in taste recognition with left insular lesion was also reported [8]. Right insula infarcts resulted in diminished sense of smell and taste [10] and deficits in ipsilateral taste recognition [8].

\section{Thalamus}

Bilateral paramedian thalamic infarction presented with a change in the quality of odor and taste perceptions in a patient described by Rousseaux et al. [13]. 


\section{Pons}

Hemorrhagic stroke at the level of the right tegmentum of the pons led in a patient to ipsilateral disturbance of taste on both the anterior two-thirds and posterior one-third of the tongue [11]. In another patient, an ischemic infarct of the right pons resulted in numbness on the right side of the tongue associated with lowered taste perception [5]. Right medial lemniscus stroke was also reported with impaired taste related to the anterior two-thirds of the left tongue [12].

\section{Diverse Locations}

Green et al. [14] reported 2 patients who experienced hyposmia (diminished sense of smell), anosmia (complete loss of smell), and dysgeusia (distorted taste). One patient had a minor infarct in the cerebral area of the anterior circulation (right corona radiate lacunar ischemic stroke), while the second patient had a lesion in the territory of the posterior circulation (posterior-lateral medulla oblongata). Kim and Choi [15] reported 4 patients who developed altered food preference due to decreased taste perception associated with dysgeusia after a unilateral cortical infarction involving the frontal opercular area. Onoda and Ikeda [16] reported 3 cases with gustatory disturbances following stroke. One patient had an infarct at the level of the right tegmental pontine region with gustatory disturbance ipsilateral to the lesion. The second patient had a left thalamic infarct with right-sided hypogeusia and the third patient had an infarct of the left internal capsular infarct, with gustatory disturbance contralateral to the lesion. Heckmann et al. [17] reported on 102 consecutive stroke patients with 31 patients suffering hypogeusia/ageusia. Taste deficits were associated with frontal, parietal, temporal, occipital lobes, being most frequent with frontal strokes (16 of 31 patients). Both thalamic and brainstem strokes (not further specified) equally led to taste deficits in 6 of the 31 patients.

\section{Limitation}

Although not all clinically suspected strokes are confirmed by MRI [18, 19], the most important limitation in our case report is that we could only perform CT imaging because of the MRI noncompatible cardioverter defibrillator. Therefore, we cannot exclude that we missed other small ischemic lesions that might have contributed to the bilateral ageusia, not detectable on CT imaging.

\section{Conclusion}

Stroke can lead to a loss of or an altered taste perception, a deficit that at first appears to be of lesser severity compared with classic stroke symptoms such as aphasia, hemiparesis or vision disturbances. However, taste perception is a fundamental part of a subject's quality of life. In our patient, a unilateral mesencephalic lesion caused bilateral ageusia. The literature reported multiple lesion sites associated with ageusia, foremost the insular cortex, the thalamus, and the pons; a mesencephalic lesion as in our case was not reported so far. A careful examination of taste function is mandatory in each stroke patient. Further research, especially lesion studies are necessary to fully understand the complex gustatory pathway. 


\section{Statement of Ethics}

No approval was required.

\section{Disclosure Statement}

The authors have no conflict of interest to declare.

\section{References}

1 Woschnagg H, Stöllberger C, Finsterer J: Loss of taste is loss of weight. Lancet 2002;359:891

12 Landis BN, Welge-Luessen A, Brämerson A, Bende M, Mueller CA, Nordin S, Hummel T: "Taste Strips" a rapid, lateralized, gustatory bedside identification test based on impreg- nated filter papers. J Neurol 2009;256:242-248.

3 Kobal G, Hummel T, Sekinger B, Barz S, Roscher S, Wolf S: “Sniffin’ Sticks:” screening of olfactory performance. Rhinology 1996;34:222-226.

4 Hummel T, Sekinger B, Wolf S, Pauli E, Kobal G: "Sniffin' Sticks:” olfactory performance assessed by the combined testing of odor identification, odor discrimination and olfactory threshold. Chem Senses $1997 ; 22: 39-52$.

-5 Landis BN, Leuchter I, San Millan Ruiz D, Lacroix JS, Landis T: Transient hemiageusia in cerebrovascular lateral pontine lesions. J Neurol Neurosurg Psychiatry 2006;77:680-683.

6 Cereda C, Ghika J, Maeder P, Bogousslavsky J: Strokes restricted to the insular cortex. Neurology 2002;59:1950-1955.

7 Metin B, Melda B, Birsen I: Unusual clinical manifestation of a cerebral infarction restricted to the insulate cortex. Neurocase 2007;13:94-96.

-8 Pritchard TC, Macaluso DA, Eslinger PJ: Taste perception in patients with insular cortex lesions. Behav Neurosci 1999;113:663-671.

-9 Dutta TM, Josiah AF, Cronin CA, Wittenberg GF, Cole JW: Altered taste and stroke: a case report and literature review. Top Stroke Rehabil 2013;20:78-86.

10 Moo L, Wityk RJ: Olfactory and taste dysfunction after bilateral middle cerebral artery stroke. J Stroke Cerebrovasc Dis 1999;8:353-354.

11 Nakajima Y, Utsumi H, Takahashi H: Ipsilateral disturbance of taste due to pontine haemorrhage. J Neurol 1983;229:133-136.

12 Sunada I, Akano Y, Yamamoto S, Tashiro T: Pontine haemorrhage causing disturbance of taste. Neuroradiology 1995;37:659.

13 Rousseaux M, Muller P, Gahide I, Mottin Y, Romon M: Disorders of smell, taste, and food intake in a patient with a dorsomedial thalamic infarct. Stroke 1996;27:2328-2330.

14 Green TL, McGregor LD, King KM: Smell and taste dysfunction following minor stroke: a case report. Can J Neurosci Nurs 2008;30:10-13.

15 Kim JS, Choi S: Altered food preference after cortical infarction: Korean style. Cerebrovasc Dis 2002;13:187-191.

16 Onoda K, Ikeda M: Gustatory disturbance due to cerebrovascular disorder. Laryngoscope 1999;109:123-128.

17 Heckmann JG, Stossel C, Lang CJ, Neundorfer B, Tomandl B, Hummel T: Taste disorders in acute stroke: a prospective observational study on taste disorders in 102 stroke patients. Stroke 2005;36:16901694.

18 Makin SD, Doubal FN, Dennis MS, Wardlaw JM: Clinically confirmed stroke with negative diffusionweighted imaging magnetic resonance imaging: longitudinal study of clinical outcomes, stroke recurrence, and systematic review. Stroke 2015;46:3142-3148.

19 Buchholz DW: Clinically probable brainstem stroke presenting primarily as dysphagia and nonvisualized by MRI. Dysphagia 1993;8:235-238. 


\section{Case Reports in Neurology}

\begin{tabular}{l|l}
\hline Case Rep Neurol 2018;10:60-65 \\
\hline DOI: 10.1159/000487299 & $\begin{array}{l}\text { C } 2018 \text { The Author(s). Published by S. Karger AG, Basel } \\
\text { www.karger.com/crn }\end{array}$ \\
\hline
\end{tabular}

Saleh et al.: Bilateral Ageusia and Tongue Anesthesia Following Unilateral Brainstem Infarct: A Case Report with a Brief Review of the Literature

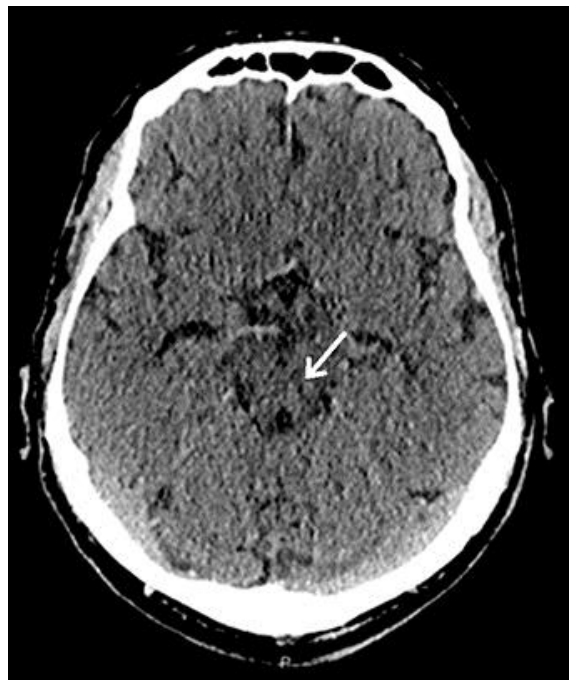

Fig. 1. The native CT scan of the brain revealed a subtle oval hypodensity on the left side in the mesencephalon (arrow).
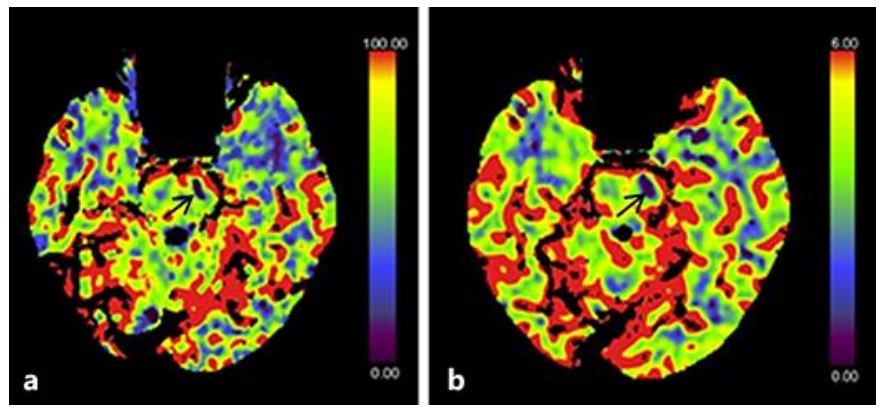

Fig. 2. CT perfusion demonstrating focal perfusion deficit with reduced cerebral blood flow (a) and cerebral blood volume (b).
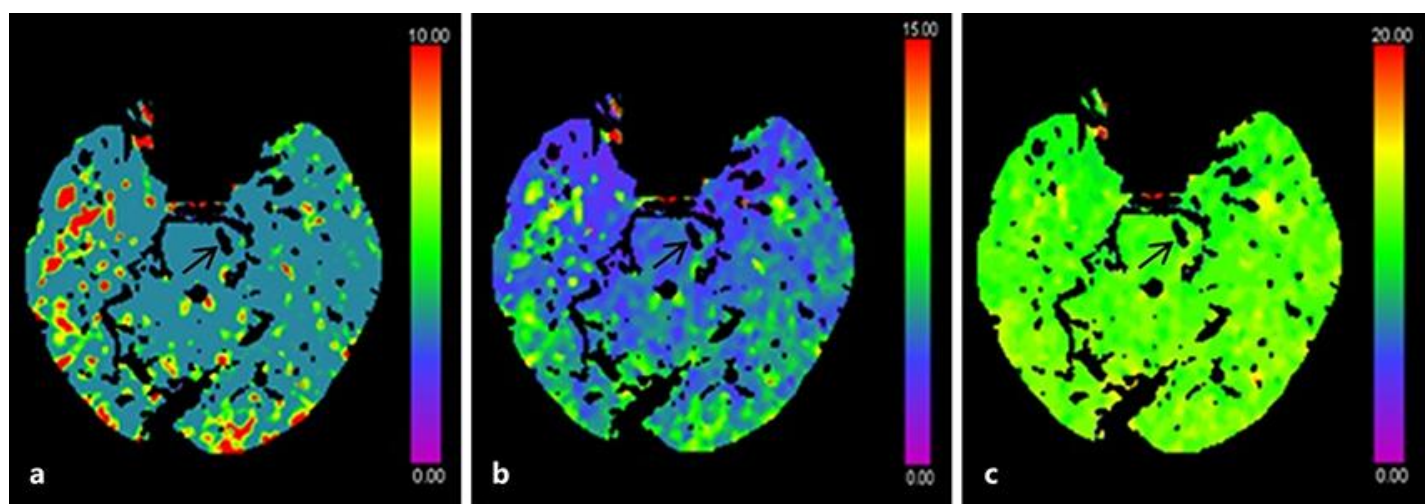

Fig. 3. Prolonged mean transit time (a), time to drain (b) and time to peak (c) indicative of a focal brainstem ischemia. 